\title{
Further validation of the Japanese version of the Multidimensional Assessment of Interoceptive Awareness
}

Haruo Fujino $12^{*}$ (1)

\begin{abstract}
Objective: The Multidimensional Assessment of Interoceptive Awareness (MAIA) is a validated measure to assess interoceptive awareness. Although an earlier study evaluated the Japanese version of the MAIA, it did not examine the measure's test-retest reliability and the data fit of the factor structure. This study aims to further validate the Japanese version of the MAIA.

Results: In this cross-sectional study, 268 Japanese individuals participated. They completed the Japanese version of the MAIA and concurrent validity measures. The test-retest reliability of the Japanese version's subscales ranged from adequate to high (intra-class coefficients $=0.76-0.85$ ). Confirmatory factor analysis demonstrated that the Japanese six-factor structure had a good fit in Japanese. The MAIA subscales were moderately associated with scores on the Body Awareness Scale $(\mathrm{rho}=0.25-0.49)$. The results indicated high test-retest reliability and further confirmed the validity of the six-factor structure of the Japanese version of the MAIA. Hence, the Japanese version of the MAIA is a useful measure to assess interoceptive awareness in the Japanese population.
\end{abstract}

Keywords: Body awareness, Body-mind, Interoceptive awareness, Validation studies, Interoception

\section{Introduction}

Recent studies have focused on interoceptive awareness as an important factor affecting the adaptivity of psychological functioning. Interoception and body awareness are cardinal features of the therapeutic process in bodymind therapies and body-oriented psychotherapy [1-6]. Interoception is a multifaceted construct that includes interoceptive awareness, attention, detection, accuracy (sensitivity), and sensibility [7-12], whereas body awareness represents perceptual and conscious awareness of subjective physical experience [12]. There is substantial overlap between interoception and body awareness. Several studies have provided conceptual models that dysfunction in interoceptive processing; these are linked to mental disorders, suggesting the interoception's

\footnotetext{
*Correspondence: finoh@oita-u.ac.jp

${ }^{1}$ Department of Special Needs Education, Oita University, 700 Dannoharu, Oita 870-1192, Japan

Full list of author information is available at the end of the article
}

mediating role in psychiatric and neurodevelopmental disorders [10, 13-15]. Two clinical studies suggested that interoceptive awareness mediates therapeutic changes in mindfulness interventions [16, 17]; however, further research is required to clarify interoception's adaptive role in mental health and mind-body interventions. Accordingly, a validated multidimensional measure is required to investigate the different aspects of interoceptive awareness.

Although several self-report measures to assess interoceptive and/or body awareness are available, they assess only very limited aspects (e.g., negative aspects) of the concept, which may not capture the complex nature of interoceptive awareness [12]. The Multidimensional Assessment of Interoceptive Awareness (MAIA) questionnaire is designed to assess interoceptive awareness [18]. It has a multidimensional construct (eight dimensions) that reflects the multidimensional nature of interoceptive awareness, as suggested by earlier studies $[12,19]$. Additionally, the MAIA has been 
used to assess changes in interoceptive awareness in interventional studies $[17,20]$.

Originally, the MAIA was developed in English; subsequently, translated versions were developed in many languages (https:/osher.ucsf.edu/maia/). An evaluation of the validity and reliability of the MAIA's Japanese version using exploratory factor analysis demonstrated that the MAIA's original factor structure was not replicated in a Japanese sample [21]. Shoji and colleagues suggested that the MAIA's Japanese version has a sixfactor structure, with several items loaded on other factors unlike the original structure [21]; however, the model fit of the six-factor and original eight-factor structures has not been examined for the MAIA's Japanese version. Additionally, the test-retest reliability, which was not examined by the previous validation study, should be confirmed with the longitudinal evaluation of interoceptive awareness. Hence, further validation is required to evaluate the psychometric properties of the Japanese version. Accordingly, this study aims to examine the validity of a six-factor model of the MAIA in a Japanese sample.

\section{Main text \\ Methods \\ Participants}

In this study, 268 participants aged 18-27 years (mean age $=19.6$; standard deviation, $\mathrm{SD}=1.3$ ) were recruited. Most of them were students at three institutions in Japan (Oita University, Osaka University, and Hiramatsu Oita College of Rehabilitation) from January 2017. Approximately half the total number of participants comprised women $(\mathrm{n}=141,53 \%)$. In addition to the aforementioned 268, 78 participants aged $18-31$ years (mean age $=19.0$, $\mathrm{SD}=1.8$ ) were recruited to examine test-retest reliability. Seventy-eight participants completed the MAIA 2 weeks ( \pm 1 week) after the first evaluation. The minimum number of participants required for this study was determined using the COnsensus-based Standards for the selection of health Measurement Instruments (COSMIN) checklist criteria $(7 \times 32$ [number of items]) [22].

All the participants were notified of the study's purpose and methods, and assured that their privacy would be protected. Once they agreed to participate by completing a written consent form, they were administered the questionnaires. Subsequently, on receiving the completed questionnaires, the research assistant checked them for missing values.

This study was approved by the Oita University Faculty of Education Research Ethics Committee (28-009). Further, it was conducted in accordance with the World Medical Association's Declaration of Helsinki.

\section{Measures}

Multidimensional Assessment of Interoceptive Awareness (MAIA) The MAIA is a 32-item self-report questionnaire that assesses the eight dimensions of interoceptive awareness on a 6-point Likert scale (0 [never] to 5 [always]) [18]. The MAIA comprises the following eight independent domains of interoceptive awareness: "Noticing," the awareness of one's physical sensations (four items); "NotDistracting," the tendency to not use distraction to avoid discomfort (three items); "Not-Worrying," the tendency to avoid worrying about uncomfortable physical sensations (three items); "Attention regulation," the ability to control one's attention to inner bodily sensations (seven items); "Emotional awareness," the ability to understand specific body sensations as physical responses to emotions (five items); "Self-regulation," the ability to regulate psychological distress using mindful attention to inner bodily sensations (four items); "Body listening," the tendency to actively listen to the body to gain insight (three items); and "Trusting," the experience of one's body as safe and trustworthy (three items). The score for each scale ranges from 0 to 5 , which is calculated by averaging the scores of the scale's individual items. Higher scores indicate higher ability or tendency in each domain. Finally, the study used the MAIA's Japanese-translated version [21].

\section{Body Awareness Scale}

The Body Awareness Scale (BAS) is a 20-item self-report measure that assesses body awareness and experience of physical sensations; it comprises four subscales [23]. We used two of its subscales, "Awareness of Bodily Feeling" and "Actual Bodily Feeling," to confirm the MAIA's concurrent validity. Whereas the first subscale assesses the tendency of being aware of inner physical sensations, the second assesses the ability to feel one's physical feelings as they truly are. The Cronbach's alpha values for the current sample's subscales were 0.78 (Awareness of Bodily Feeling) and 0.65 (Actual Bodily Feeling).

\section{Statistical analysis}

We examined the internal consistency of the MAIA's Japanese version using item-scale correlation and Cronbach's alpha calculation. A Cronbach's alpha higher than 0.70 indicated good internal consistency. Further, we evaluated the MAIA's test-retest reliability by using an intra-class correlation coefficient (ICC) with a two-way, mixed-effects model. An ICC higher than 0.70 indicated good test-retest reliability.

Subsequently, using confirmatory factor analysis, we examined the model fit of the two models for the MAIA. The first model was the original eight-factor model that used all the 32 items of the MAIA, and the second 
model was the Japanese six-factor model that used the 25 items of the MAIA suggested by Shoji and colleagues [21]. The model fit was evaluated using Chi square statistics, the comparative fit index (CFI), the Tucker-Lewis Index (TLI), the standardized root mean square residual (SRMR), and the root mean square error of approximation (RMSEA). We considered nonsignificant Chi square statistics, CFI and TLI $\geq 0.95$, and SRMR and RMSEA $\leq 0.08$ to indicate a good fit. Further, we estimated the MAIA's factor structure using a polychoric correlation matrix and the diagonally weighted least squares method, which is designed for ordinal data [24].

Additionally, we evaluated concurrent validity by considering associations between related concepts. The earlier validation study used the Five Facet Mindfulness Questionnaire, Difficulties in Emotion Regulation Scale, and Pain Catastrophizing Scale as concurrent measures [21]. In this study, we examined the association between the MAIA subscales and body awareness, which was not examined in the previous study. Corresponding scales were determined according to the measured concept in each domain prior to the analysis (Additional file 1: Table S1).

Statistical analyses were performed using R 3.4 .1 statistical software ( $\mathrm{R}$ Core Team, Vienna, Austria). The significance level was set at a two-tailed $p<0.05$ after Bonferroni correction to achieve an overall family-wise error rate of 0.05 for the associations between the MAIA and the BAS subscales (12 tests). The corrected $p$ values are reported in "Results" section.

\section{Results}

Descriptive statistics for the MAIA items and reliability

Seven of the eight subscales adequately satisfied the criteria for high internal consistency and test-retest reliability. The item-total correlations were adequate in all subscales (0.70-0.88). Further, the Cronbach's alphas values of all the MAIA subscales except one were high, ranging from 0.72 (Not-Distracting) to 0.87 (Attention Regulation) (Table 1). Similarly, the ICC of each subscale was high (0.74-0.87). However, the Not-Worrying subscale, which was included in the original structure, had poor internal consistency $(\alpha=0.32)$ and slightly less testretest reliability $(\mathrm{ICC}=0.68)$.

\section{Model fit and concurrent validity of the Japanese MAIA}

To evaluate model fit, the original eight-factor and Japanese six-factor constructs were evaluated using confirmatory factor analysis. The original eight-factor model showed generally acceptable fit in all fit indices except RMSEA (0.084), whereas the six-factor model showed acceptable fit in all goodness-of-fit indices (CFI $=0.981$, $\mathrm{TLI}=0.978, \mathrm{SRMR}=0.067$, RMSEA $=0.078[90 \%$ confidence interval 0.071-0.085]) (Table 2). Further, the standardized coefficients of all the item loadings were sufficiently high (0.57-0.90).

We examined the correlations between the six subscales of the Japanese MAIA and the BAS subscales. Most of the MAIA subscales were moderately correlated with the conceptually corresponding BAS subscales (Table 3). The Not-Distracting subscale was independent

Table 1 Descriptive statistics, internal consistency indices, and test-retest reliability of the MAIA subscales

\begin{tabular}{|c|c|c|c|c|c|c|c|}
\hline Subscale & No. of items & Median & Mean & SD & $\begin{array}{l}\text { Item-total } \\
\text { correlation }\end{array}$ & $\begin{array}{l}\text { Cronbach's } \\
\text { alpha }\end{array}$ & ICC (95\% Cl) \\
\hline \multicolumn{8}{|l|}{ Original 8-factor structure } \\
\hline Noticing & 4 & 2.8 & 2.69 & 0.98 & $0.71-0.79$ & 0.74 & $0.74(0.63-0.83)$ \\
\hline Not-distracting & 3 & 2.7 & 2.64 & 1.00 & $0.74-0.82$ & 0.72 & $0.76(0.65-0.84)$ \\
\hline Not-worrying & 3 & 2.0 & 2.19 & 0.87 & $0.47-0.74$ & 0.32 & $0.68(0.54-0.78)$ \\
\hline Attention regulation & 7 & 2.6 & 2.49 & 0.85 & $0.70-0.83$ & 0.87 & $0.85(0.77-0.90)$ \\
\hline Emotional awareness & 5 & 2.8 & 2.70 & 1.00 & $0.72-0.87$ & 0.85 & $0.87(0.80-0.91)$ \\
\hline Self-regulation & 4 & 2.8 & 2.76 & 0.89 & $0.73-0.81$ & 0.74 & $0.86(0.79-0.91)$ \\
\hline Body listening & 3 & 2.0 & 2.13 & 1.04 & $0.85-0.86$ & 0.82 & $0.81(0.71-0.87)$ \\
\hline Trusting & 3 & 3.0 & 2.83 & 1.01 & $0.79-0.88$ & 0.80 & $0.78(0.68-0.86)$ \\
\hline \multicolumn{8}{|c|}{ Japanese 6-factor structure } \\
\hline Noticing $^{a}$ & $5^{a}$ & 2.6 & 2.66 & 0.94 & $0.70-0.79$ & 0.78 & $0.85(0.77-0.90)$ \\
\hline Not $=$ distracting & 3 & 2.7 & 2.64 & 1.00 & $0.74-0.82$ & 0.72 & $0.76(0.65-0.84)$ \\
\hline Attention regulation & 7 & 2.6 & 2.49 & 0.85 & $0.70-0.83$ & 0.87 & $0.85(0.77-0.90)$ \\
\hline Emotional awareness $^{a}$ & $3^{a}$ & 3.0 & 2.81 & 1.12 & $0.86-0.88$ & 0.84 & $0.82(0.73-0.88)$ \\
\hline Body listening $^{a}$ & $4^{a}$ & 2.3 & 2.31 & 0.99 & $0.74-0.83$ & 0.82 & $0.82(0.73-0.88)$ \\
\hline Trusting & 3 & 3.0 & 2.83 & 1.01 & $0.79-0.88$ & 0.80 & $0.78(0.68-0.86)$ \\
\hline
\end{tabular}

a The items included in the subscale differ from the original 
Table 2 Fit indices from the confirmatory factor analysis of the Japanese MAIA

\begin{tabular}{llllllll}
\hline Model & Chi square & df & $\mathbf{p}$ & CFI & TLI & SRMR & RMSEA (90\% Cl) \\
\hline $\begin{array}{c}\text { Original 8-factor } \\
\text { model (32 items) }\end{array}$ & 1262.8 & 436 & $<0.001$ & 0.973 & 0.969 & 0.073 & $0.084(0.079-0.090)$ \\
$\begin{array}{c}\text { Japanese 6-factor } \\
\text { model (25 items) }\end{array}$ & 684.2 & 159 & $<0.001$ & 0.981 & 0.978 & 0.067 & $0.078(0.071-0.085)$ \\
\hline
\end{tabular}

Table 3 Spearman's correlation coefficients between the MAIA subscales (Japanese six-factor model) and the Body Awareness Scale

\begin{tabular}{lcl}
\hline & $\begin{array}{l}\text { Awareness of bodily } \\
\text { Feeling }\end{array}$ & $\begin{array}{l}\text { Actual bodily } \\
\text { Feeling }\end{array}$ \\
\hline Noticing & $0.49^{* * *}$ & $0.35^{* * *}$ \\
Not-distracting & $-0.06^{* * *}$ & 0.10 \\
Attention regulation & $0.35^{* * *}$ & $0.44^{* * *}$ \\
Emotional awareness & $0.28^{* * *}$ & $0.25^{* * *}$ \\
Body listening & $0.35^{* * *}$ & $0.33^{* * *}$ \\
Trusting & $0.29^{* * *}$ & $0.44^{* * *}$ \\
\hline
\end{tabular}

*** $p<0.001$

of the BAS scores. Finally, we confirmed 9 of the 10 predefined hypotheses on the correlations (Additional file 1: Table S1).

\section{Discussion}

This study evaluated the model fit of the original eightfactor and Japanese six-factor structures of the MAIA in a Japanese sample. The results conformed to the original Japanese validation study, suggesting a six-factor model fit for the Japanese MAIA and the potential necessity of modifying its original subscales.

The properties of the Japanese MAIA were similar to those of the original English and other translated versions, except for one subscale. Although seven of the eight subscales of the MAIA's Japanese version showed good internal consistency and reliability, the Not-Worrying subscale had low internal consistency and slightly low test-retest reliability, which is in line with previous validation studies. These studies showed a low internal consistency for the Not-Distracting and Not-Worrying subscales [18, 20, 25-28]. In the current study, the NotDistracting subscale had good internal consistency, whereas the Not-Worrying subscale showed poor internal consistency. This trend has been observed in clinical samples, as well (e.g., those with current or past low back pain or with eating disorders) [29, 30], suggesting psychometric issues in the construct.

In the confirmatory factor analysis, the Japanese sixfactor model showed good fit to the current sample, whereas the original eight-factor model did not satisfy the good-fit criterion for RMSEA $(\leq 0.08)$. The results suggest the necessity of making minor modifications (e.g., deletion or addition of items) to the original eightfactor model to validate the MAIA scale in cross-cultural contexts $[20,25,26,28]$. Since an earlier study suggested that conceptual and cultural differences may affect the construct of the MAIA [21], the findings imply that subjective aspects of interoceptive awareness are affected by the conceptual framework of a culture or population.

The previous Japanese validation study showed moderate associations between the MAIA and concurrent measures [21]. In the current sample, we further demonstrated a moderate association between the MAIA and a body awareness measure. Although most of the pre-specified hypotheses were confirmed, the MAIA's Not-Distracting subscale was not correlated with the BAS subscales. Accordingly, the concept measured by the Not-Distracting subscale may be independent compared to the other subscales $[28,31]$. The original MAIA was recently updated due to the problems in some scales, as described in "Discussion" section. Currently, the updated 37-item MAIA version 2, which was unavailable at the time of this study's data collection, is available [32]. Hence, the use of MAIA version 2 in future studies is recommended.

In conclusion, this study showed high test-retest reliability and reconfirmed the validity of the Japanese version of the MAIA's six-factor structure. Hence, the Japanese version of the MAIA is a reliable and validated measure to assess interoceptive awareness in the Japanese population.

\section{Limitations}

The study has several limitations. This study did not examine the associations between improvement in interoceptive awareness and other adaptive abilities or functioning of individuals. The study's findings have limited applicability since the characteristics of the study's sample are nearly identical to those of a sample used by an earlier study [21]. Additionally, the low Cronbach's alpha for the BAS subscale Actual Bodily Feeling may limit the correlations that can be identified with the Not-distracting subscale of the MAIA. 


\section{Additional file}

Additional file 1: Table S1. Hypotheses tested for concurrent validity. The summary of the hypotheses tested for concurrent validity.

\section{Abbreviations}

BAS: Body Awareness Scale; CFI: comparative fit index; COSMIN: COnsensusbased Standards for the selection of health Measurement Instruments; ICC: intraclass correlation coefficient; MAIA: Multidimensional Assessment of Interoceptive Awareness; RMSEA: root mean square error of approximation; SRMR: standardized root mean square residual; TLI: Tucker-Lewis Index.

\section{Acknowledgements}

The author acknowledge Dr. Osamu Imura for his advice and support for data collection.

\section{Authors' contributions}

HF designed the study, collected, analyzed and interpreted the data, and wrote the manuscript. The author read and approved the final manuscript.

\section{Funding}

This work was supported in part by research grants from the Mental Health Okamoto Memorial Foundation (Ken-45). The funders had no role in the study design, data collection and analyses, decision to publish, or preparation of the manuscript.

\section{Availability of data and materials}

The datasets used and analyzed during the current study are available from the corresponding author upon reasonable request to the corresponding author.

\section{Ethics approval and consent to participate}

This study was conducted in accordance with the ethical standards set forth in the 1964 Declaration of Helsinki and its later amendments and was approved by the Oita University Faculty of Education Research Ethics Committee (28-009). All of the participants were explained the purpose and methods of the study, and assured about the protection of their privacy. They were administered the questionnaires after agreeing to participate by completing a written consent form.

\section{Consent for publication}

Not applicable.

\section{Competing interests}

The author declares that he has no competing interests.

\section{Author details}

${ }^{1}$ Department of Special Needs Education, Oita University, 700 Dannoharu, Oita 870-1192, Japan. ${ }^{2}$ Graduate School of Human Sciences, Osaka University, Suita, Japan.

Received: 4 July 2019 Accepted: 10 August 2019

Published online: 20 August 2019

\section{References}

1. Fujino $\mathrm{H}$. Body awareness and mental health: a body psychotherapy case study. Body Mov Dance Psychother. 2016;11:249-62.

2. Gyllensten AL, Hansson L, Ekdahl C. Patient experiences of basic body awareness therapy and the relationship with the physiotherapist. J Bodyw Mov Ther. 2003:7:173-83.

3. Hedlund L, Gyllensten AL. The experiences of basic body awareness therapy in patients with schizophrenia. J Bodyw Mov Ther. 2010;14:245-54

4. Landsman-Dijkstra JJ, van Wijck R, Groothoff JW. The long-term lasting effectiveness on self-efficacy, attribution style, expression of emotions and quality of life of a body awareness program for chronic a-specific psychosomatic symptoms. Patient Educ Couns. 2006:60:66-79.
5. Levy Berg A, Sandahl C, Bullington J. Patients' perspective of change processes in affect-focused body psychotherapy for generalised anxiety disorder. Body Mov Dance Psychother. 2010;5:151-69.

6. Röhricht F, Papadopoulos N, Holden S, Clarke T, Priebe S. Clinical effectiveness and therapeutic processes of body psychotherapy in chronic schizophrenia_an open clinical trial. Arts Psychother. 2011;38:196-203.

7. Farb N, Daubenmier J, Price CJ, Gard T, Kerr C, Dunn BD, et al. Interoception, contemplative practice, and health. Front Psychol. 2015;6:763.

8. Garfinkel SN, Critchley HD. Interoception, emotion and brain: new insights link internal physiology to social behaviour. Commentary on: "Anterior insular cortex mediates bodily sensibility and social anxiety" by Terasawa et al. (2012). Soc Cogn Affect Neurosci. 2013;8:231-4.

9. Garfinkel SN, Seth AK, Barrett AB, Suzuki K, Critchley HD. Knowing your own heart: distinguishing interoceptive accuracy from interoceptive awareness. Biol Psychol. 2015;104:65-74.

10. Herbert BM, Pollatos $\mathrm{O}$. The body in the mind: on the relationship between interoception and embodiment. Top Cogn Sci. 2012;4:692-704.

11. Khalsa SS, Adolphs R, Cameron OG, Critchley HD, Davenport PW, Feinstein JS, et al. Interoception and mental health: a Roadmap. Biol Psychiatry Cogn Neurosci Neuroimag. 2018;3:501-13.

12. Mehling WE, Gopisetty V, Daubenmier J, Price CJ, Hecht FM, Stewart A. Body awareness: construct and self-report measures. PLOS ONE. 2009;4:e5614

13. Krautwurst $\mathrm{S}$, Gerlach $\mathrm{AL}$, Witthoft M. Interoception in pathological health anxiety. J Abnorm Psychol. 2016;125:1179-84.

14. Murphy J, Brewer R, Catmur C, Bird G. Interoception and psychopathology: a developmental neuroscience perspective. Dev Cogn Neurosci. 2017;23:45-56

15. Paulus MP, Stein MB. Interoception in anxiety and depression. Brain Struct Funct. 2010;214:451-63.

16. de Jong M, Lazar SW, Hug K, Mehling WE, Holzel BK, Sack AT, et al. Effects of mindfulness-based cognitive therapy on body awareness in patients with chronic pain and comorbid depression. Front Psychol. 2016;7:967.

17. Mehling WE, Chesney MA, Metzler TJ, Goldstein LA, Maguen S, Geronimo C, et al. A 12-week integrative exercise program improves self-reported mindfulness and interoceptive awareness in war veterans with posttraumatic stress symptoms. J Clin Psychol. 2018;74:554-65.

18. Mehling WE, Price C, Daubenmier JJ, Acree M, Bartmess E, Stewart A. The Multidimensional Assessment of Interoceptive Awareness (MAIA). PLoS ONE. 2012;7:e48230.

19. Mehling WE, Wrubel J, Daubenmier JJ, Price CJ, Kerr CE, Silow T, et al. Body Awareness: a phenomenological inquiry into the common ground of mind-body therapies. Philos Ethics Humanit Med. 2011;6:6.

20. Bornemann B, Herbert BM, Mehling WE, Singer T. Differential changes in self-reported aspects of interoceptive awareness through 3 months of contemplative training. Front Psychol. 2014;5:1504.

21. Shoji M, Mehling WE, Hautzinger $M$, Herbert BM. Investigating multidimensional interoceptive awareness in a Japanese Population: validation of the Japanese MAIA-J. Front Psychol. 2018;9:1855.

22. Terwee CB, Mokkink LB, Knol DL, Ostelo RW, Bouter LM, de Vet HC. Rating the methodological quality in systematic reviews of studies on measurement properties: a scoring system for the COSMIN checklist. Qual Life Res. 2012;21:651-7

23. Fujino $\mathrm{H}$. Effects of Dohsa-hou relaxation on body awareness and psychological distress. Jpn Psychol Res. 2012;54:388-99.

24. Li CH. Confirmatory factor analysis with ordinal data: comparing robust maximum likelihood and diagonally weighted least squares. Behav Res Methods. 2016:48:936-49.

25. Baranauskas M, Grabauskaitè A, Griškova-Bulanova I. Psychometric characteristics of Lithuanian version of Multidimensional Assessment of Interoceptive Awareness (MAIA). Neurologijos seminarai. 2016;20:202-6.

26. Cali G, Ambrosini E, Picconi L, Mehling WE, Committeri G. Investigating the relationship between interoceptive accuracy, interoceptive awareness, and emotional susceptibility. Front Psychol. 2015;6:1202. https://doi. org/10.3389/fpsyg.2015.01202.

27. Lin FL, Hsu CC, Mehling W, Yeh ML. Translation and psychometric testing of the chinese version of the multidimensional assessment of interoceptive awareness. J Nurs Res. 2017;25:76-84.

28. Valenzuela-Moguillansky C, Reyes-Reyes A. Psychometric properties of the multidimensional assessment of interoceptive awareness (MAIA) in a Chilean population. Front Psychol. 2015;6:120. 
29. Brown TA, Berner LA, Jones MD, Reilly EE, Cusack A, Anderson LK, et al. Psychometric evaluation and norms for the Multidimensional Assessment of Interoceptive Awareness (MAIA) in a clinical eating disorders sample. Eur Eat Disord Rev. 2017;25:411-6.

30. Mehling WE, Daubenmier J, Price CJ, Acree M, Bartmess E, Stewart AL. Self-reported interoceptive awareness in primary care patients with past or current low back pain. J Pain Res. 2013;6:403-18.

31. Hanley AW, Mehling WE, Garland EL. Holding the body in mind: interoceptive awareness, dispositional mindfulness and psychological wellbeing. J Psychosom Res. 2017;99:13-20.
32. Mehling WE, Acree $M$, Stewart A, Silas J, Jones A. The multidimensional assessment of interoceptive awareness, version 2 (MAIA-2). PLOS ONE. 2018;13:e0208034.

\section{Publisher's Note}

Springer Nature remains neutral with regard to jurisdictional claims in published maps and institutional affiliations.
Ready to submit your research? Choose BMC and benefit from:

- fast, convenient online submission

- thorough peer review by experienced researchers in your field

- rapid publication on acceptance

- support for research data, including large and complex data types

- gold Open Access which fosters wider collaboration and increased citations

- maximum visibility for your research: over $100 \mathrm{M}$ website views per year

At BMC, research is always in progress.

Learn more biomedcentral.com/submissions 